\title{
A NOTE ON THE QUASI-STATIONARY DISTRIBUTION OF THE SHIRYAEV MARTINGALE ON THE POSITIVE HALF-LINE*
}

\author{
ALEKSEY S. POLUNCHENKO ${ }^{\dagger}$, SERVET MARTÍNEZ ${ }^{\ddagger}$, AND JAIME SAN MARTÍN $\ddagger$
}

\begin{abstract}
We obtain a closed-form formula for the quasi-stationary distribution of the classical Shiryaev martingale diffusion considered on the positive half-line $[A,+\infty)$ with $A>0$ fixed; the state space's left endpoint is assumed to be the killing boundary. The formula is obtained analytically as the solution of the appropriate singular Sturm-Liouville problem; the latter was first considered in [7, Section 7.8.2], but has heretofore remained unsolved.
\end{abstract}

Key words. Quasi-stationary distribution, Shiryaev martingale, Whittaker functions

AMS subject classifications. 60J60, 60F99, 33C15

1. Introduction and problem formulation. This work centers around the stochastic process known as the Shiryaev martingale. Specifically, the latter is defined as the solution $\left(X_{t}\right)_{t \geqslant 0}$ of the stochastic differential equation (SDE)

$$
d X_{t}=d t+X_{t} d B_{t} \text { with } X_{0} \triangleq x_{0} \geqslant 0 \text { fixed, }
$$

where $\left(B_{t}\right)_{t \geqslant 0}$ is standard Brownian motion (i.e., $\mathbb{E}\left[d B_{t}\right]=0, \mathbb{E}\left[\left(d B_{t}\right)^{2}\right]=d t$, and $\left.B_{0}=0\right)$; the initial value $X_{0}=x_{0} \geqslant 0$ is sometimes also referred to as the headstart. The name "Shiryaev martingale" has apparently been introduced in [7, Section 7.8.2] and is due to two reasons. The first reason is to acknowledge the fact that equation (1) was first arrived at and extensively studied by Prof. A.N. Shiryaev in his fundamental work (see $[34,35])$ in the area of quickest change-point detection where the process $\left(X_{t}\right)_{t \geqslant 0}$ has become known as the Shiryaev-Roberts detection statistic, and it is one of the "central threads" in the field. See also, e.g., [29, 36, 14, 3, 31, 30]. The second reason is that the time-homogeneous Markov diffusion $\left(X_{t}\right)_{t \geqslant 0}$ is easy to see to have the martingale property $\mathbb{E}\left[X_{t}-x_{0}-t\right]=0$ for any $t \geqslant 0$, i.e., the process $\left\{X_{t}-x_{0}-t\right\}_{t \geqslant 0}$ is a zero-mean martingale.

The SDE (1) is a special case of the more general SDE $d Z_{t}=\left(a Z_{t}+b\right) d t+Z_{t} d B_{t}$ where $Z_{0}=z_{0} \in \mathbb{R}, a \in \mathbb{R}$, and $b \in \mathbb{R}$ are fixed; note that the process $\left\{Z_{t}-z_{0}-t\right\}_{t \geqslant 0}$ is not a zero-mean martingale, unless $a=0$ and $b=1$. The process $\left(Z_{t}\right)_{t \geqslant 0}$ is sometimes called the Shiryaev process or the Shiryaev diffusion, for it, too, was arrived at and studied by Prof. A.N. Shiryaev in [34, 35] in the context of quickest change-point detection. However, the Kolmogorov [18] forward and backward equations corresponding to the Shiryaev process $\left(Z_{t}\right)_{t \geqslant 0}$ have also arisen independently in areas far beyond quickest change-point detection, notably in mathematical physics, and, more recently, in mathematical finance. By way of example, in mathematical physics, the authors of $[27,8]$ dealt with $Z_{t}$ interpreting it as the position at time $t$ of a particle moving around in an inhomogeneous environment driven by a combination of random forces (e.g., thermal noise). Financial significance of the Shiryaev process

\footnotetext{
${ }^{*}$ Submitted to the editors DATE.

Funding: The effort of A. S. Polunchenko was partially supported by the Simons Foundation via a Collaboration Grant in Mathematics under Award \# 304574.

${ }^{\dagger}$ Department of Mathematical Sciences, State University of New York at Binghamton, Binghamton, New York, USA (aleksey@binghamton.edu, http://people.math.binghamton.edu/aleksey).

${ }^{\ddagger}$ CMM-DIM, UMI-CNRS 2807, Basal PBF-03, Universidad de Chile, Santiago, Chile (smartine@dim.uchile.cl, jsanmart@dim.uchile.cl).
} 
has been understood, e.g., in [15, 11, 21], in relation to so-called arithmetic Asian options where $Z_{t}$ represents the option's price at time $t$. Moreover, the Shiryaev process also proved useful as a stochastic interest rate model. See, e.g., [15, 39, 10, 26, 9]. Last but not least, the Shiryaev diffusion is also of interest in itself as a stochastic process, especially due to its close connection to geometric Brownian motion (which $Z_{t}$ is when $\left.b=0\right)$. See, e.g., [42, 43, 11, $\left.12,33,28,32\right]$.

The Shiryaev process - whether $\left(X_{t}\right)_{t \geqslant 0}$ or $\left(Z_{t}\right)_{t \geqslant 0}$-is typically considered either on a compact subset of the real line, or on the entire real line, or on one of the two halflines $(-\infty, 0]$ or $[0,+\infty)$. This work draws attention to the case when the state space for $\left(X_{t}\right)_{t \geqslant 0}$ is the set $[A,+\infty)$ where $A>0$ is given. Specifically, we shall assume that $\left(X_{t}\right)_{t \geqslant 0}$ is started off a point inside the interval $[A,+\infty)$, i.e., $X_{0} \triangleq x_{0} \in[A,+\infty)$, and then the process is let continue until it hits the lower boundary $A>0$ whereat the process is terminated. The question of interest is the process' long-term behavior, conditional that the process is not killed. More formally, consider the stopping time

$$
S_{A} \triangleq \inf \left\{t \geqslant 0: X_{t}=A\right\} \text { such that } \inf \{\varnothing\}=+\infty,
$$

where $A>0$ is given. The specific aim of this paper is to obtain an exact closed-form formula for the Shiryaev martingale's quasi-stationary distribution. Specifically, this distribution is defined as

(3) $Q_{A}(x) \triangleq \lim _{t \rightarrow+\infty} \mathbb{P}\left(X_{t} \leqslant x \mid S_{A}>t\right)$ with $q_{A}(x) \triangleq \frac{d}{d x} Q_{A}(x)$, where $x \in[A,+\infty)$,

and $X_{0} \triangleq x_{0}>A$ and $A>0$ are preset. The existence of this distribution has been previously asserted in [7, Section 7.8.2], which, to the best of our knowledge, is also where the very problem of finding either $Q_{A}(x)$ or $q_{A}(x)$ in a closed form was first formulated, but has heretofore remained unsolved. The solution we obtain analytically in Section 3 elucidates the general theory of quasi-stationary phenomena associated with killed one-dimensional Markov diffusions set forth in the seminal work of Mandl [23] and then further developed by Collet, Martínez and San Martín in [6, $24,25,7]$; see also [4]. The obtained formulae for $Q_{A}(x)$ or $q_{A}(x)$ complement those previously found by Polunchenko [31] for the case when the Shiryaev martingale is restricted to the interval $[0, A]$ with $A>0$ fixed.

2. Preliminaries. For notational brevity, we shall henceforth omit the subscript " $A$ " in " $Q_{A}(x)$ " as well as in " $q_{A}(x)$ ", unless the dependence on $A$ is noteworthy. Also, for technical convenience and without loss of generality, we shall primarily deal with $q(x)$ rather than with $Q(x)$.

It is has already been established in the literature (see, e.g., [7, Section 7.8.2] or $[23,4])$ that $q(x)$, formally defined in (3), is the solution of a certain boundary-value problem composed of a second-order ordinary differential equation (ODE) considered on the half line $(A,+\infty)$, a normalization constraint, a set of boundary conditions along with a square-integrability restriction. Specifically, the ODE - which we shall refer to as the master equation - is of the form

$$
\frac{1}{2} \frac{d^{2}}{d x^{2}}\left[x^{2} q(x)\right]-\frac{d}{d x}[q(x)]=-\lambda q(x), \quad x \in(A,+\infty),
$$

where $\lambda \geqslant 0$ is the smallest eigenvalue of the differential operator

$$
\mathscr{D} \triangleq \frac{1}{2} \frac{\partial^{2}}{\partial x^{2}} x^{2}-\frac{\partial}{\partial x}
$$


which is the infinitesimal generator of the Shiryaev diffusion $\left(X_{t}\right)_{t \geqslant 0}$ governed by SDE (1); we remark that the nonnegativity of $\lambda$ is not an assumption, and it will be formally asserted below that, in fact, $\lambda \in(0,1 / 8]$. It goes without saying that $\lambda$ is dependent on $A$, and, wherever necessary, we shall emphasize this dependence via the notation $\lambda_{A}$.

The relation

$$
\lambda_{A}=-\lim _{t \rightarrow+\infty}\left\{\frac{1}{t} \mathbb{P}\left(S_{A}>t\right)\right\}
$$

where $S_{A}$ is the stopping time defined in (2), lends $\lambda_{A}$ the flavor of the killing rate for $\left(X_{t}\right)_{t \geqslant 0}$; cf. [7]. In fact, in [7], the relation (6) is used to argue that $\lambda_{A}$ is an increasing function of $A>0$. Moreover, it is also established in [7, Section 7.8.2] that $\lambda_{A}=1 / 8$ is guaranteed for $A \geqslant e^{3} \approx 20.0855369$. We shall see in the next section that $\lambda_{A}=1 / 8$ is attained for much smaller values of $A$, namely for $A \geqslant A^{*} \approx 1.265857361$ with $A^{*}$ being the solution of a certain transcendental equation.

The normalization constraint that $q(x)$ is to satisfy is the natural requirement

$$
\int_{A}^{+\infty} q(x) d x=1
$$

which is merely the statement that $q(x)$, as a pdf supported on $[A,+\infty)$, must integrate to unity over its entire support.

It is easily checked that the state space's lower boundary $x=A>0$ is a regular absorbing boundary, and the upper boundary $x=+\infty$ is a natural boundary. Therefore, there is only one boundary condition to impose on $q(x)$, and this condition is at $x=A$, and it is as follows:

$$
q(A)=0
$$

which, in "differential equations speak", is a Dirichlet-type boundary condition. While no boundary condition is required at $x=+\infty$, there is a certain square-integrability restriction required to hold for $q(x)$ around $x=+\infty$. This restriction will be explained below.

Subject to the absorbing boundary condition (8), the normalization constraint (7), and the square-integrability restriction yet to be discussed, the master equation (4) is a Sturm-Liouville problem. It is a singular problem, for the domain, i.e., the interval $[A,+\infty)$, is unbounded. The singular nature of the problem affects the spectrum $\{\lambda\}$ of the corresponding operator $\mathscr{D}$ given by $(5)$. By virtue of the multiplying factor

$$
\mathfrak{m}(x) \triangleq \frac{2}{x^{2}} e^{-\frac{2}{x}}
$$

the master equation can be brought to the canonical Sturm-Liouville form

$$
\frac{1}{2} \frac{d}{d x}\left[x^{2} \mathfrak{m}(x) \frac{d}{d x} \varphi(x)\right]=-\lambda \mathfrak{m}(x) \varphi(x),
$$

where the unknown function $\varphi(x)$ is such that $q(x) \propto \mathfrak{m}(x) \varphi(x)$, i.e., $q(x)$ is a multiple of $\mathfrak{m}(x) \varphi(x)$. Hence, the operator $\mathscr{D}$ given by (5) is equivalent to the Sturm-Liouville operator

$$
\mathscr{G} \triangleq \frac{1}{2 \mathfrak{m}(x)} \frac{\partial}{\partial x} x^{2} \mathfrak{m}(x) \frac{\partial}{\partial x}
$$


with $\mathfrak{m}(x)$ given by (9). Although operators $\mathscr{G}$ and $\mathscr{D}$ are essentially formal adjoints of one another, the former is more convenient to deal with because it is in a canonical Sturm-Liouville form, so that the general theory of Sturm-Liouville operators can be readily utilized to gain preliminary insight into the spectral characteristics of $\mathscr{G}$. It is evident from (5) and (11) that $\mathscr{G}$ and $\mathscr{D}$ have the same spectra, and that their corresponding eigenfunctions differ by a factor of $\mathfrak{m}(x)$ given by (9).

The general theory of second-order differential operators or Sturm-Liouville operators (such as our operators $\mathscr{G}$ and $\mathscr{D}$ introduced above) is well-developed, and, in particular, the spectral properties of such operators are well-understood. The classical fundamental references on the subject are [38], [19], [5], [13], and [20]; for applications of the theory to diffusion processes, see, e.g., Ito and McKean [17, Section 4.11], and especially Linetsky [22] who provides a great overview of the state-of-the-art in the field considered in the context of stochastic processes. For our specific problem, the general Sturm-Liouville theory immediately establishes that the eigenfunctions $\varphi(x) \equiv \varphi(x, \lambda)$ indexed by $\lambda$ of the operator $\mathscr{G}$ given (11) form an orthonormal basis in the Hilbert space $L^{2}([A,+\infty), \mathfrak{m})$ of real-valued $\mathfrak{m}(x)$-measurable, square-integrable (with respect to the $\mathfrak{m}(x)$ measure) functions defined on the interval $[A,+\infty)$ equipped with the "mo $(x)$ "-weighted inner product:

$$
\langle f, g\rangle_{\mathfrak{m}} \triangleq \int_{A}^{+\infty} \mathfrak{m}(x) f(x) g(x) d x .
$$

More specifically, the foregoing means that if $\lambda^{(i)}$ and $\lambda^{(j)}$ are any two eigenvalues of $\mathscr{G}$, and $\varphi\left(x, \lambda^{(i)}\right)$ and $\varphi\left(x, \lambda^{(j)}\right)$ are the corresponding eigenfunctions, then

$$
\int_{A}^{+\infty} \mathfrak{m}(x) \varphi\left(x, \lambda^{(i)}\right) \varphi\left(x, \lambda^{(j)}\right) d x=\mathbb{1}_{\{i=j\}},
$$

where $\varphi\left(x, \lambda^{(i)}\right)$ and $\varphi\left(x, \lambda^{(j)}\right)$ are each assumed to be of unit "length", in the sense that $\left\|\varphi\left(\cdot, \lambda^{(i)}\right)\right\|=1=\left\|\varphi\left(\cdot, \lambda^{(j)}\right)\right\|$, with the "length" defined as

$$
\|\varphi(\cdot, \lambda)\|^{2} \triangleq \int_{A}^{+\infty} \mathfrak{m}(x) \varphi^{2}(x, \lambda) d x .
$$

We are now in a position to state the square-integrability restriction on $q(x)$ : it is the requirement that $\|\varphi(\cdot, \lambda)\|<+\infty$ for the very same $\lambda$ that is present in (4).

To gain further insight into the spectral properties of the operator $\mathscr{G}$ we turn to the work of Linetsky [22] who introduces three mutually exclusive Spectral Categories of Sturm-Liouville operators, and establishes easy-to-use criteria to determine which specific category a given Sturm-Liouville operator falls under. The classification is based on the nature of the corresponding domain boundaries, viz. whether the boundaries are oscillatory or non-oscillatory. The classification criteria are given by [22, Theorem 3.3, p. 248]. Specifically, by appealing to [22, Theorem 3.3(ii), p. 248] it is straightforward to verify that our Sturm-Liouville problem belongs to Spectral Category II introduced in [22, Theorem 3.2(ii), p. 246]. This means that the spectrum $\{\lambda\}$ of the operators $\mathscr{G}$ and $\mathscr{D}$ is simple and nonnegative, i.e., all $\lambda \geqslant 0$. Moreover, the spectrum is purely absolutely continuous in $(1 / 8,+\infty)$ where the $1 / 8$ is the spectrum cutoff point. Finally, since [22, Theorem 3.3(ii), p. 248] shows that $x=+\infty$ is a non-oscillatory boundary, the operators $\mathscr{G}$ and $\mathscr{D}$ may also have a finite set of simple eigenvalues inside the interval $[0,1 / 8]$, and these eigenvalues are determined entirely by the Dirichlet boundary condition (8) or equivalently $\varphi(A, \lambda)=0$. 
We conclude this section with a remark that $\lambda=0$ is not an option. Indeed, observe that in this case the function $\varphi(x, 0)=K$ for some constant $K$ does solve (10) and is square-integrable with respect to the $\mathfrak{m}(x)$ measure given by (9). However, the function $q(x) \propto \mathfrak{m}(x)$, although does solve (4), is possible to normalize in accordance with the normalization constraint (7) only if $K \neq 0$. Yet, if $K \neq 0$, then the absorbing boundary condition (8) is impossible to fulfil, because, in view of (9), no nontrivial multiple of $\mathfrak{m}(x)$ can be turned into zero at any finite $x=A>0$. On the other hand, if $K=0$, then the absorbing boundary condition (8) is trivially satisfied, but the normalization constraint (7) can never be. Therefore $\lambda=0$ is not an eigenvalue of the operator $\mathscr{D}$ given by (5), and its spectrum $\{\lambda\}$ lies entirely inside the interval $(0,1 / 8]$. This was previously conjectured in [7, Section 7.8.2]. The strict positivity of the smallest eigenvalue of $\mathscr{D}$ enables us to enjoy all of the results already obtained in [7, Section 7.8.2], starting from the very fact that $q(x)$ must exist. In the next section this distribution will be expressed in a closed form through analytic solution of the corresponding Sturm-Liouville problem.

3. The quasi-stationary distribution formulae. The plan now is to fix $A>$ 0 and solve the master equation (4) analytically and thereby recover both $q_{A}(x)$ and $Q_{A}(x)$ in a closed form for all $x \in[A,+\infty)$. To that end, it is easier to deal with the equivalent equation (10), and the first step to treat it is to apply the change of variables

$$
x \mapsto u=u(x)=\frac{2}{x}, \text { so that } u \mapsto x=x(u)=\frac{2}{u} \text { and } \frac{d x}{x}=-\frac{d u}{u},
$$

along with the substitution

$$
\varphi(x) \mapsto \varphi(u) \triangleq \frac{v(u)}{\sqrt{\mathfrak{m}(u)}} \propto \frac{1}{u} e^{\frac{u}{2}} v(u),
$$

to bring the equation to the form

$$
v_{u u}(u)+\left\{-\frac{1}{4}+\frac{1}{u}+\frac{1 / 4-\xi^{2} / 4}{u^{2}}\right\} v(u)=0
$$

where

$$
\xi \equiv \xi(\lambda) \triangleq \sqrt{1-8 \lambda} \text { so that } \lambda \equiv \lambda(\xi)=\frac{1}{8}\left(1-\xi^{2}\right),
$$

and $\xi \in[0,1)$ on account of $\lambda \in(0,1 / 8]$ concluded in the previous section. The restriction $\xi \in[0,1)$ will be invoked repeatedly throughout the remainder of this section. The change of variables (13) and the substitution (14) were devised to treat a similar Sturm-Liouville problem in the closely related work of Polunchenko [31]; see also [21] and [32]. We also remark that equation (15) is symmetric with respect to the sign of $\xi$, i.e., one could also define $\xi$ as $\xi \triangleq-\sqrt{1-8 \lambda}$. However, as will become clear shortly, this ambiguity in the definition of $\xi$ has no effect on the sought quasi-stationary density $q(x)$ whatsoever.

The obtained equation (15) is a particular case of the classical Whittaker [40] equation

$$
w_{z z}(z)+\left\{-\frac{1}{4}+\frac{a}{z}+\frac{1 / 4-b^{2}}{z^{2}}\right\} w(z)=0
$$


where $w(z)$ is the unknown function of $z \in \mathbb{C}$, and $a, b \in \mathbb{C}$ are specified parameters. This self-adjoint homogeneous second-order ODE is well-known in mathematical physics as well as in mathematical finance. Its two linearly independent fundamental solutions are known as the Whittaker functions. These functions are special functions that take a variety of forms depending on the specific values of $a$ and $b$. The classical references on the general theory of the Whittaker equation (17) and Whittaker functions are [37] and [2]. For our purposes it will prove convenient and sufficient to deal with the Whittaker $M$ and $W$ functions, which are conventionally denoted, respectively, as $M_{a, b}(z)$ and $W_{a, b}(z)$, where the indices $a$ and $b$ are the Whittaker's equation (17) parameters.

By combining (17), (15), (14) and (13), one can now see that the general form of $\varphi(x, \lambda)$ is

$$
\varphi(x, \lambda)=x e^{\frac{1}{x}}\left\{B_{1} M_{1, \frac{1}{2} \xi(\lambda)}\left(\frac{2}{x}\right)+B_{2} W_{1, \frac{1}{2} \xi(\lambda)}\left(\frac{2}{x}\right)\right\}, x \in[A,+\infty),
$$

where $\xi(\lambda)$ is as in (16), and $B_{1}$ and $B_{2}$ are arbitrary constants such that $B_{1} B_{2} \neq 0$. It is of note that both of the two Whittaker functions involved in the obtained formula for $\varphi(x, \lambda)$ are well-defined, real-valued, and linearly independent of each other for any $\xi(\lambda) \in[0,1)$. Also, from [1, Identity 13.1.34, p. 505], i.e., from the identity

$$
W_{a, b}(z)=\frac{\Gamma(-2 b)}{\Gamma(1 / 2-b-a)} M_{a, b}(z)+\frac{\Gamma(2 b)}{\Gamma(1 / 2+b-a)} M_{a,-b}(z),
$$

where here and onward $\Gamma(z)$ denotes the Gamma function (see, e.g., [1, Chapter 6]), one can readily conclude that (18) is unaffected by the sign ambiguity in the definition (16) of $\xi \equiv \xi(\lambda)$.

The obvious next step is to recall that $q(x) \propto \mathfrak{m}(x) \varphi(x, \lambda)$, where $\mathfrak{m}(x)$ is given by (9), and, in view of (18), conclude that the quasi-stationary density $q(x)$ has the general form

$$
q(x)=\frac{1}{x} e^{-\frac{1}{x}}\left\{C_{1} M_{1, \frac{1}{2} \xi(\lambda)}\left(\frac{2}{x}\right)+C_{2} W_{1, \frac{1}{2} \xi(\lambda)}\left(\frac{2}{x}\right)\right\}, x \in[A,+\infty),
$$

where $C_{1}$ and $C_{2}$ are constant factors to be designed so as to make $q(x)$ satisfy the absorbing boundary condition (8) as well as the normalization constraint (7). With regard to the former, it is straightforward to see from (20) that $C_{1}$ and $C_{2}$ must satisfy the equation

$$
C_{1} M_{1, \frac{1}{2} \xi(\lambda)}\left(\frac{2}{A}\right)+C_{2} W_{1, \frac{1}{2} \xi(\lambda)}\left(\frac{2}{A}\right)=0,
$$

and it is not a degenerate equation in the sense that the Whittaker $M$ and $W$ involved in it are never zeros simultaneously, for the Whittaker $M$ and $W$ functions with the same indices and arguments (finite) may become zeros at the same time only at the origin.

To proceed, observe that

$$
\begin{aligned}
W_{a, b}(x) \sim \frac{\Gamma(-2 b)}{\Gamma(1 / 2-b-a)} & x^{\frac{1}{2}+b} e^{-\frac{1}{2} x}+ \\
& +\frac{\Gamma(2 b)}{\Gamma(1 / 2+b-a)} x^{\frac{1}{2}-b} e^{-\frac{1}{2} x} \text { as } x \rightarrow 0+,
\end{aligned}
$$


which is a direct consequence of (19) and the asymptotics

$$
M_{a, b}(x) \sim x^{\frac{1}{2}+b} e^{-\frac{1}{2} x} \text { as } x \rightarrow 0+,
$$

established, e.g., in [41, Section 16.1, p. 337]. Recalling yet again that $\xi \in[0,1)$, it follows from (20) and the asymptotics (22) and (23) that $q(x)$ is $\mathfrak{m}(x)$-measurable for any $\lambda \in(0,1 / 8]$. Hence, let us fix $\lambda \in(0,1 / 8]$ and attempt to normalize $q(x)$ given by (20) in accordance with the normalization constraint (7). To do so, we turn to [16, Integral 7.623.3, p. 832] which states that

$$
\begin{aligned}
& \int_{0}^{t}(t-x)^{c-1} x^{a-1} e^{-\frac{1}{2} x} M_{a+c, b}(x) d x= \\
&= \frac{\Gamma(c) \Gamma(a+b+1 / 2)}{\Gamma(a+b+c+1 / 2)} t^{a+c-1} e^{-\frac{1}{2} t} M_{a, b}(t), \\
& \quad \text { provided } \Re(a+b)>-1 / 2 \text { and } \Re(c)>0,
\end{aligned}
$$

and to $[16$, Integral 7.623 .8$, p. 833$]$ which states that

$$
\begin{gathered}
\int_{0}^{1}(1-x)^{c-1} x^{a-c-1} e^{-\frac{1}{2} t x} W_{a, b}(t x) d x= \\
=\Gamma(c) e^{-\frac{t}{2}} \sec [(a-c-b) \pi]\left\{\sin (c \pi) \frac{\Gamma(a-c+b+1 / 2)}{\Gamma(2 b+1)} M_{a-c, b}(t)+\right. \\
\left.+\cos [(a-b) \pi] W_{a-c, b}(t)\right\}, \\
\text { provided } 0<\Re(c)<\Re(a)-|\Re(b)|+1 / 2,
\end{gathered}
$$

and obtain

$$
\int_{A}^{+\infty} q(x) d x=e^{-\frac{1}{A}}\left\{C_{1} \frac{2}{\xi(\lambda)+1} M_{0, \frac{1}{2} \xi(\lambda)}\left(\frac{2}{A}\right)-C_{2} W_{0, \frac{1}{2} \xi(\lambda)}\left(\frac{2}{A}\right)\right\}
$$

whence

$$
C_{1} \frac{2}{\xi(\lambda)+1} M_{0, \frac{1}{2} \xi(\lambda)}\left(\frac{2}{A}\right)-C_{2} W_{0, \frac{1}{2} \xi(\lambda)}\left(\frac{2}{A}\right)=e^{\frac{1}{A}}
$$

which is another equation that the constants $C_{1}$ and $C_{2}$ involved in (20) are to satisfy, and, just as (21), this equation is also nondegenerate. Therefore, by solving equations (21) and (26) for $C_{1}$ and $C_{2}$, and then plugging them over into (20), we arrive at the formula

$$
\begin{aligned}
q(x)=\frac{C}{x} e^{-\frac{1}{x}\left\{W_{1, \frac{1}{2} \xi(\lambda)}\right.}\left(\frac{2}{A}\right) M_{1, \frac{1}{2} \xi(\lambda)}\left(\frac{2}{x}\right)- \\
\left.-M_{1, \frac{1}{2} \xi(\lambda)}\left(\frac{2}{A}\right) W_{1, \frac{1}{2} \xi(\lambda)}\left(\frac{2}{x}\right)\right\} \mathbb{1}_{\{x \in[A,+\infty)\}},
\end{aligned}
$$

where $A>0$ and $\xi(\lambda)$ is as in (16) with $\lambda \in(0,1 / 8]$ arbitrary, and $C$ is the normalizing 
factor given by

$$
\begin{aligned}
C \triangleq e^{\frac{1}{A}} /\left\{W_{0, \frac{1}{2} \xi(\lambda)}\right. & \left(\frac{2}{A}\right) M_{1, \frac{1}{2} \xi(\lambda)}\left(\frac{2}{A}\right)+ \\
& \left.+\frac{2}{\xi(\lambda)+1} M_{0, \frac{1}{2} \xi(\lambda)}\left(\frac{2}{A}\right) W_{1, \frac{1}{2} \xi(\lambda)}\left(\frac{2}{A}\right)\right\} .
\end{aligned}
$$

It turns out that the above formula for $C$ can be substantially simplified with the aid of the identity

$$
W_{0, b}(z) M_{1, b}(z)+\frac{1}{b+1 / 2} M_{0, b}(z) W_{1, b}(z)=\frac{z}{\sqrt{\pi}} \frac{2^{2 b}}{b+1 / 2} \Gamma(1+b),
$$

which can be established through an astute use of various properties of the Whittaker functions. From this identity it is easy to see that (28) can be reduced down to

$$
C \triangleq \sqrt{\pi} \frac{A}{4} e^{\frac{1}{A}} \frac{\xi(\lambda)+1}{2^{\xi(\lambda)}} / \Gamma\left(\frac{1}{2} \xi(\lambda)+1\right),
$$

whence, recalling again that $\xi(\lambda) \in[0,1)$, it can be concluded at once that $C>0$ for any $A>0$; the positivity of the normalizing factor $C$ is equivalent to saying that the integral of the general $q(x)$ given by (27) with respect to $x$ over the interval $[A,+\infty)$ is always positive.

We are now in a position to make the following claim.

Lemma 3.1. For any fixed $A>0$ and arbitrary $\lambda \in(0,1 / 8]$, the function

$$
\begin{aligned}
q(x)=\frac{C}{x} e^{-\frac{1}{x}\left\{W_{1, \frac{1}{2} \xi(\lambda)}\right.}\left(\frac{2}{A}\right) M_{1, \frac{1}{2} \xi(\lambda)}\left(\frac{2}{x}\right)- \\
\left.-M_{1, \frac{1}{2} \xi(\lambda)}\left(\frac{2}{A}\right) W_{1, \frac{1}{2} \xi(\lambda)}\left(\frac{2}{x}\right)\right\} \mathbb{1}_{\{x \in[A,+\infty)\}},
\end{aligned}
$$

with $\xi(\lambda) \in[0,1)$ as in (16) and $C>0$ given by either (28) or (29), solves the master equation (4), and satisfies the absorbing boundary condition (8) as well as the normalization constraint (7). Moreover, the following definite integral identity also holds:

$$
\begin{aligned}
\int_{A}^{x} q(y) d y=1-C e^{-\frac{1}{x}\left\{W_{0, \frac{1}{2} \xi(\lambda)}\left(\frac{2}{x}\right) M_{1, \frac{1}{2} \xi(\lambda)}\left(\frac{2}{A}\right)+\right.} \\
\left.\quad+\frac{2}{\xi(\lambda)+1} M_{0, \frac{1}{2} \xi(\lambda)}\left(\frac{2}{x}\right) W_{1, \frac{1}{2} \xi(\lambda)}\left(\frac{2}{A}\right)\right\}
\end{aligned}
$$

for any $x \in[A,+\infty)$.

Proof. We only need to show (31). To that end, the result can be obtained by integrating (27) with respect to $x$ and evaluating the integral with the aid of the definite integral identities (24) and (25).

The only question that has not yet been addressed is that of actually finding $\lambda$. To that end, recall that $\varphi(x, \lambda)$ given by (18) must be square-integrable with 
respect to the $\mathfrak{m}(x)$ measure given by (9). More concretely, this means that $\varphi(x, \lambda)$ must be such that $\|\varphi(\cdot, \lambda)\|<+\infty$ where the norm $\|\varphi(\cdot, \lambda)\|$ is defined in (12). Due to the Whittaker $W$ and $M$ functions' near-origin behavior given by (22) and (23), respectively, the function $\varphi(x, \lambda)$ is not square-integrable near $x=+\infty$, unless either $\lambda=1 / 8$ or $B_{2}=0$ in the right-hand side of (18). As a result, we are to distinguish two separate cases: (a) $\lambda \in(0,1 / 8)$ and $B_{2}=0$ in (18), and (b) $\lambda=1 / 8$.

Let us first suppose that $\lambda \in(0,1 / 8)$. Since in this case we must set $B_{2}=0$ in (18) to achieve $\|\varphi(\cdot, \lambda)\|<+\infty$, it follows that we must also set $C_{2}=0$ in (20). If $C_{2}=0$ then from (21) it is clear that the only way for $C_{1}$ to have a nontrivial value is to demand that $\lambda$ and $A>0$ be connected via the equation

$$
M_{1, \frac{1}{2} \xi(\lambda)}\left(\frac{2}{A}\right)=0
$$

where $\xi(\lambda)$ is as in (16). Although this equation does not permit a closed form solution $\lambda \equiv \lambda_{A}$ as a function of $A>0$, it can be gleaned from [2, p. 182] that this equation does have at most one solution $\lambda \equiv \lambda_{A} \in(0,1 / 8]$ for any $A>0$. More concretely, this solution is an increasing function of $A>0$, and it ceases to exist as soon as $A>0$ reaches the value $A^{*}$ that is the solution of the equation

$$
M_{1,0}\left(\frac{2}{A^{*}}\right)=0
$$

and although this equation is also transcendental, it is easily solvable numerically with any desired accuracy, yielding $A^{*} \approx 1.265857361$.

We wrote a Mathematica script to solve equation (32) for $\lambda_{A}$ numerically. Figure 1 was obtained with the help of our Mathematica script, and it shows the behavior of $\lambda_{A}$ as a function of $A \in\left(0, A^{*}\right]$. It is clear from the figure that $\lambda_{A}(>0)$ is an increasing function of $A(>0)$ rapidly growing up to the value of $1 / 8=0.125$, which is the cutoff point of the spectrum of the operator $\mathscr{D}$ given by (5). Moreover, the figure also shows that the value of $1 / 8$ is attained by $\lambda_{A}$ at $A=A^{*} \approx 1.265857361$ where $A^{*}$ is the solution of equation (33). All this not only fulfills but also improves some of the predictions previously made in [7, Section 7.8.2], where, in particular, it was shown that $\lambda_{A}=1 / 8$ is guaranteed for $A \geqslant e^{3} \approx 20.0855369$.

We can now conclude that, if $A \in\left(0, A^{*}\right)$, then the quasi-stationary distribution's pdf and cdf are given by (30) and (31), respectively, with $\lambda_{A} \in(0,1 / 8)$ determined as the only solution of equation (32).

Let us now switch attention to the case when $\lambda=1 / 8$. From the above discussion it follows that this case takes effect for $A \geqslant A^{*} \approx 1.265857361$. The quasi-stationary distribution formulae (30) and (31) remain valid "as is", except that $\xi(\lambda)$ becomes $\xi(1 / 8)=0$

At this point we have effectively proved the following two theorems.

THEOREM 3.2. If $A^{*} \approx 1.265857361$ is the solution of the equation

$$
M_{1,0}\left(\frac{2}{A^{*}}\right)=0
$$

then for every fixed $A \in\left(0, A^{*}\right)$ the equation

$$
M_{1, \frac{1}{2} \xi(\lambda)}\left(\frac{2}{A}\right)=0 \text { with } \xi(\lambda) \triangleq \sqrt{1-8 \lambda}
$$




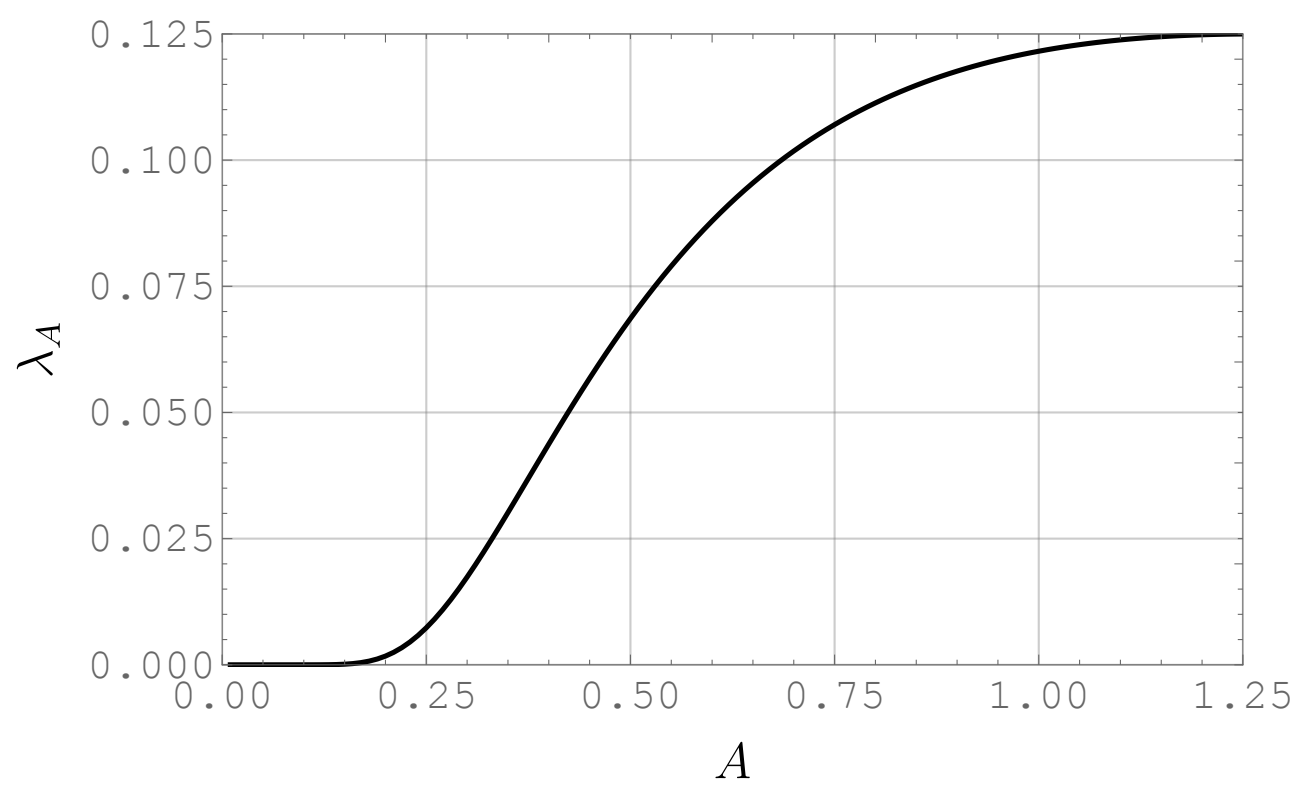

FIG. 1. Smallest eigenvalue $\lambda_{A} \in(0,1 / 8]$ of Sturm-Liouville operator $\mathscr{D}$ as a function of $A \in\left(0, A^{*}\right]$.

has exactly one solution $\lambda \equiv \lambda_{A} \in(0,1 / 8)$, and the quasi-stationary density $q_{A}(x)$ is given by

$$
q_{A}(x)=\frac{\frac{\xi(\lambda)+1}{x} e^{-\frac{1}{x}} M_{1, \frac{1}{2} \xi(\lambda)}\left(\frac{2}{x}\right)}{2 e^{-\frac{1}{A}} M_{0, \frac{1}{2} \xi(\lambda)}\left(\frac{2}{A}\right)} \mathbb{1}_{\{x \in[A,+\infty)\}}
$$

while the respective quasi-stationary $c d f Q_{A}(x)$ is given by

$$
Q_{A}(x)=\left\{\begin{array}{l}
1-\frac{e^{-\frac{1}{x}} M_{0, \frac{1}{2} \xi(\lambda)}\left(\frac{2}{x}\right)}{e^{-\frac{1}{A}} M_{0, \frac{1}{2} \xi(\lambda)}\left(\frac{2}{A}\right)}, \text { for } x \in(A,+\infty) ; \\
0, \text { otherwise. }
\end{array}\right.
$$

THEOREM 3.3. If $A^{*} \approx 1.265857361$ is the solution of the equation

$$
M_{1,0}\left(\frac{2}{A^{*}}\right)=0,
$$

then for every fixed $A \geqslant A^{*}$ the equation

$$
M_{1, \frac{1}{2} \xi(\lambda)}\left(\frac{2}{A}\right)=0 \text { with } \xi(\lambda) \triangleq \sqrt{1-8 \lambda}
$$

has no solution $\lambda \equiv \lambda_{A} \in(0,1 / 8]$ except $\lambda \equiv \lambda_{A}=1 / 8$ attained at $A=A^{*}$, and the 
quasi-stationary density $q_{A}(x)$ is given by

$$
\begin{aligned}
q_{A}(x)=\sqrt{\pi} e^{\frac{1}{A}} \frac{A}{4 x} e^{-\frac{1}{x}\left\{W_{1,0}\left(\frac{2}{A}\right)\right.} M_{1,0}\left(\frac{2}{x}\right)- \\
\left.-M_{1,0}\left(\frac{2}{A}\right) W_{1,0}\left(\frac{2}{x}\right)\right\} \mathbb{1}_{\{x \in[A,+\infty)\}},
\end{aligned}
$$

while the respective quasi-stationary $c d f Q_{A}(x)$ is given by

$$
Q_{A}(x)=\left\{\begin{aligned}
& 1-\sqrt{\pi} e^{\frac{1}{A}} \frac{A}{4} e^{-\frac{1}{x}}\left\{W_{0,0}\left(\frac{2}{x}\right) M_{1,0}\left(\frac{2}{A}\right)+\right. \\
&\left.+2 M_{0,0}\left(\frac{2}{x}\right) W_{1,0}\left(\frac{2}{A}\right)\right\}, \text { for } x \in(A,+\infty)
\end{aligned}\right.
$$

0 , otherwise.

Theorems 3.2 and 3.3 both easily follow directly from Lemma 3.1 and the discussion preceding it. It is also worth pointing out that, in view of (28) and (29), formulae (34) and (35) each permit a different expression, similar to that of formulae (36) and (37), respectively. The possibility of formulae (34) and (35) taking the form akin to that of formulae (36) and (37), respectively, is an indication that the quasi-stationary distribution is a smooth, continuous function of $A>0$.

As complicated as the obtained formulae (34)-(35) and (36)-(37) may seem, they are all perfectly amenable to numerical evaluation, meaning that the quasi-stationary distribution's pdf and cdf can all be evaluated numerically to within any desired accuracy, for any $A>0$. To illustrate this point, we implemented the formulae in a Mathematica script, and used it to perform a few numerical experiments each corresponding to a specific value of $A>0$. The obtained results are presented next.

Figures 2, 3, and 4 depict the quasi-stationary pdf $q_{A}(x)$ and the corresponding $\operatorname{cdf} Q_{A}(x)$ as functions of $1 / x \in[0,1 / A]$ for $A=1 / 10, A=1 / 2$, and for $A=1$, respectively. We stress that the $x$-axis scale is not $x \in[A,+\infty)$ but is $1 / x \in[0,1 / A]$. This is intentional, and is done to achieve finiteness of the domain of the quasistationary distribution. On the flip side, however, this transformation effectively reverses the direction of the $x$-axis, which is why the $\operatorname{cdf} Q_{A}(x)$ appears as a decreasing function of $x$ : it is not, as long as one keeps in mind that the $x$-axis is the reciprocal of $x$. It is also of note that $A=\{1 / 10,1 / 2,1\}$ are all smaller than $A^{*} \approx 1.265857361$, so that $\lambda_{A} \in(0,1 / 8)$ and the corresponding pdf $q(x)$ and $\operatorname{cdf} Q(x)$ are given by Theorem 3.2.

Figures 5, 6, and 7 show the quasi-stationary pdf $q(x)$ and $\operatorname{cdf} Q(x)$ for $A=2$, $A=5$, and $A=10$, respectively. Since $A=\{2,5,10\}$ are all higher than $A^{*} \approx$ 1.265857361 , it follows that $\lambda=1 / 8$ and the corresponding pdf $q(x)$ and $\operatorname{cdf} Q(x)$ are given by Theorem 3.3.

To draw a line under the entire paper, we remark that formulae (27)-(29) actually give a whole family of quasi-stationary densities $q_{A}(x)$ indexed by $\lambda \in\left(0, \lambda_{A}\right)$ where $\lambda_{A} \in(0,1 / 8]$ is the "bottom" of the spectrum of the operator $\mathscr{D}$ defined in (5). Put another way, for any fixed $A>0$ and any fixed $\lambda \in\left(0, \lambda_{A}\right)$, the function $q_{A}(x)$ given by formulae (27)-(29) is "legitimate" pdf supported on $[A,+\infty)$, because it is nonnegative for any $x \in[A,+\infty)$ and integrates to unity over the interval $[A,+\infty)$. Indeed, first note that if $\lambda \in\left(0, \lambda_{A}\right)$, then $q_{A}(x)$ must be different from zero for all 


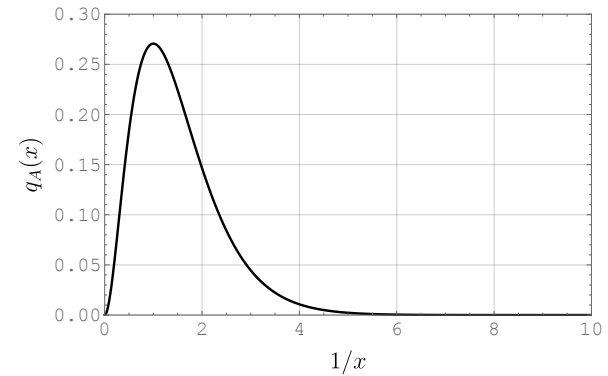

(a) $q_{A}(x)$.

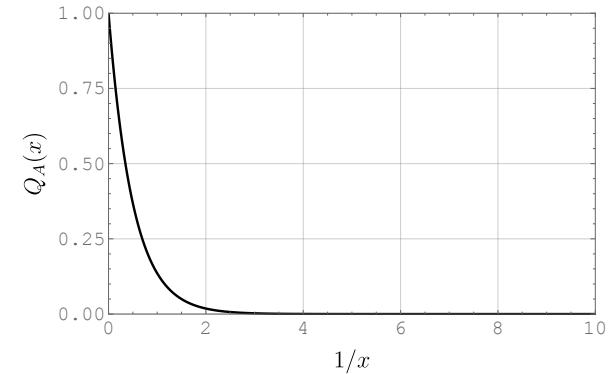

(b) $Q_{A}(x)$.

FIG. 2. Quasi-stationary distribution's pdf $q_{A}(x)$ and cdf $Q_{A}(x)$ as functions of $1 / x$ for $A=1 / 10$.

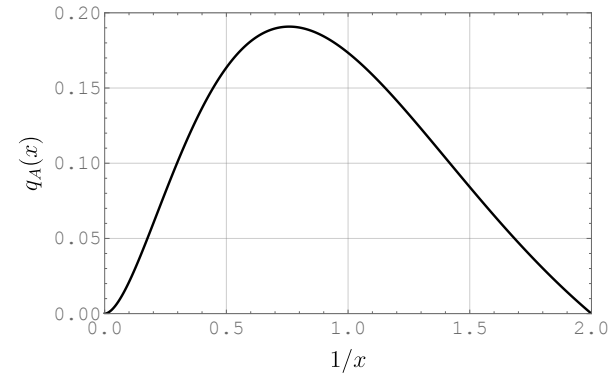

(a) $q_{A}(x)$.

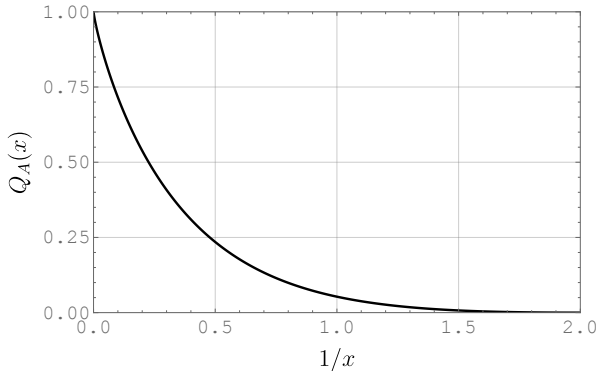

(b) $Q_{A}(x)$.

FIG. 3. Quasi-stationary distribution's $p d f q_{A}(x)$ and $c d f Q_{A}(x)$ as functions of $1 / x$ for $A=1 / 2$.

$x>A$, for otherwise $\lambda_{A}$ would not be the smallest eigenvalue. Therefore $q_{A}(x)$ is either positive or negative for all $x>A$. To see that $q_{A}(x)$ cannot be negative, observe that from (31) we have

$$
\begin{aligned}
\int_{A}^{+\infty} q(y) d y=1-C \lim _{x \rightarrow 0+}\left\{e^{-x}\right. & {\left[W_{0, \frac{1}{2} \xi(\lambda)}(2 x) M_{1, \frac{1}{2} \xi(\lambda)}\left(\frac{2}{A}\right)+\right.} \\
& \left.\left.+\frac{2}{\xi(\lambda)+1} M_{0, \frac{1}{2} \xi(\lambda)}(2 x) W_{1, \frac{1}{2} \xi(\lambda)}\left(\frac{2}{A}\right)\right]\right\},
\end{aligned}
$$

where we recall that $C>0$ is a constant (independent of $x$ but dependent on $A$ ) given by either (28) or (29). However, according to (22) and (23), the limit in the right-hand side of the foregoing formula is zero, because $\xi(\lambda) \in[0,1)$ whenever $\lambda \epsilon$ $(0,1 / 8]$, as can be easily seen from (16). Thus $q_{A}(x)$ given by (27)-(29) integrates to one over the interval $[A,+\infty)$. This necessitates that the sign maintained by $q_{A}(x)$ in the interior of this interval be positive. We therefore arrive at the curious conclusion: formulae (27)-(29) yield a "legitimate" quasi-stationary pdf $q_{A}(x)$ for any $\lambda \in\left(0, \lambda_{A}\right) \subset(0,1 / 8]$. However, while $\varphi(x, \lambda)$ given (18) is still an eigenfunction of $\mathscr{D}$, it satisfies the square-integrability condition only for $\lambda=\lambda_{A}$, i.e., $\|\psi(\cdot, \lambda)\|<+\infty$ is false, unless $\lambda=\lambda_{A}$. The existence such a continuum of quasi-stationary distributions was also previously predicted in [7, Section 7.8.2]; see also [7, Corollary 6.19, p. 144, and Theorem 6.34, p. 157]. 


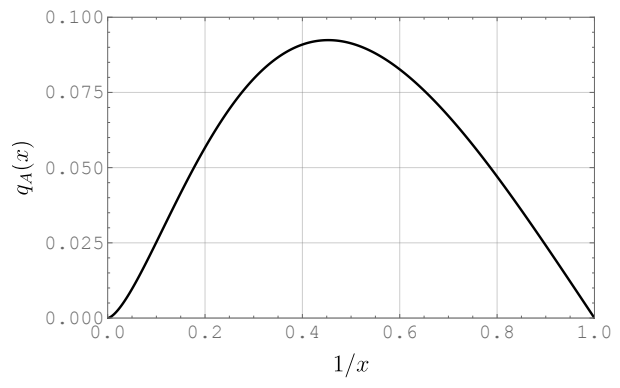

(a) $q_{A}(x)$.

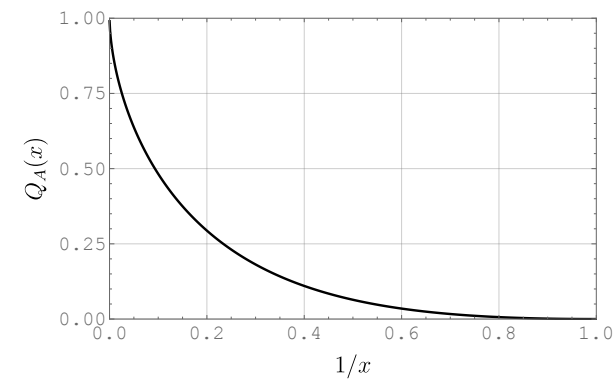

(b) $Q_{A}(x)$.

FIG. 4. Quasi-stationary distribution's pdf $q_{A}(x)$ and cdf $Q_{A}(x)$ as functions of $1 / x$ for $A=1$.

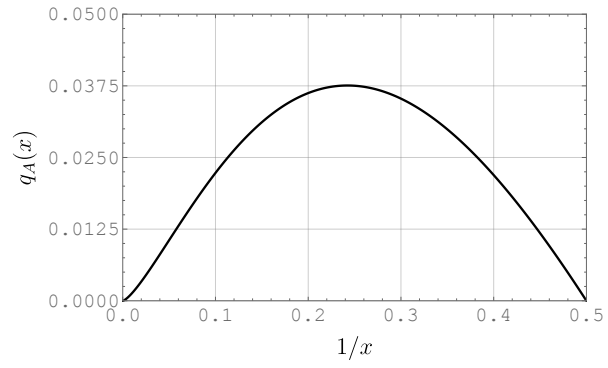

(a) $q_{A}(x)$.

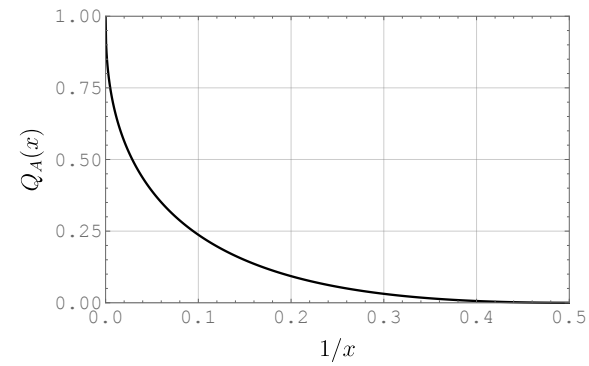

(b) $Q_{A}(x)$.

FIG. 5. Quasi-stationary distribution's $p d f q_{A}(x)$ and $c d f Q_{A}(x)$ as functions of $1 / x$ for $A=2$.

Acknowledgments. The authors would like to thank Dr. E.V. Burnaev of the Kharkevich Institute for Information Transmission Problems, Russian Academy of Sciences, Moscow, Russia, and Prof. A.N. Shiryaev of the Steklov Mathematical Institute, Russian Academy of Sciences, Moscow, Russia, for their interest in and attention to this work.

\section{REFERENCES}

[1] M. Abramowitz and I. Stegun, eds., Handbook of Mathematical Functions with Formulas, Graphs, and Mathematical Tables, vol. 55 of Applied Mathematics Series, United States National Bureau of Standards, tenth ed., 1964.

[2] H. Buchnolz, The Confluent Hypergeometric Function, vol. 15 of Springer Tracts in Natural Philosophy, Springer-Verlag, New York, NY, 1969. Translated from German into English by $\mathrm{H}$. Lichtblau and K. Wetzel.

[3] E. V. Burnaev, E. A. Feinberg, and A. N. Shiryaev, On asymptotic optimality of the second order in the minimax quickest detection problem of drift change for Brownian motion, Theory of Probability and Its Applications, 53 (2009), pp. 519-536, https://doi.org/10. 1137/S0040585X97983791.

[4] P. Cattiaux, P. Collet, A. Lambert, S. Martínez, S. Méléard, and J. S. Martín, Quasistationary distributions and diffusion models in population dynamics, Annals of Probability, 37 (2009), pp. 1926-1969, https://doi.org/10.1214/09-AOP451.

[5] E. A. Coddington and N. Levinson, Theory of Ordinary Differential Equations, McGrawHill, New York, NY, 1955.

[6] P. Collet, S. Martínez, and J. S. Martín, Asymptotic laws for one-dimensional diffusions conditioned to nonabsorption, Annals of Probability, 23 (1995), pp. 1300-1314, https:// doi.org/10.1214/aop/1176988185. 


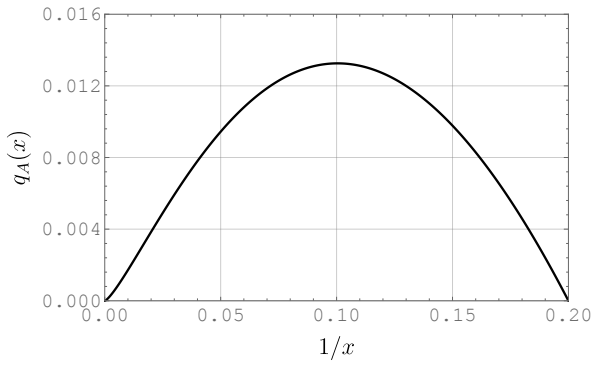

(a) $q_{A}(x)$

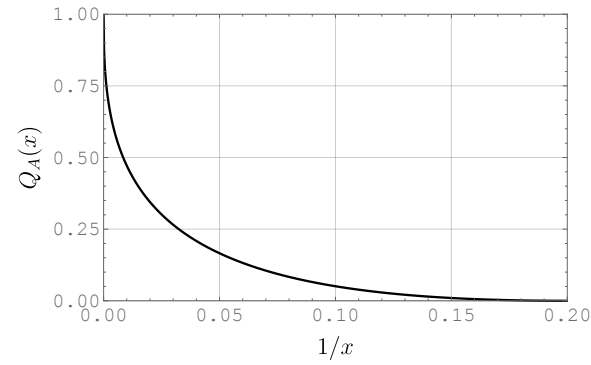

(b) $Q_{A}(x)$.

FIG. 6. Quasi-stationary distribution's $p d f q_{A}(x)$ and cdf $Q_{A}(x)$ as functions of $1 / x$ for $A=5$.

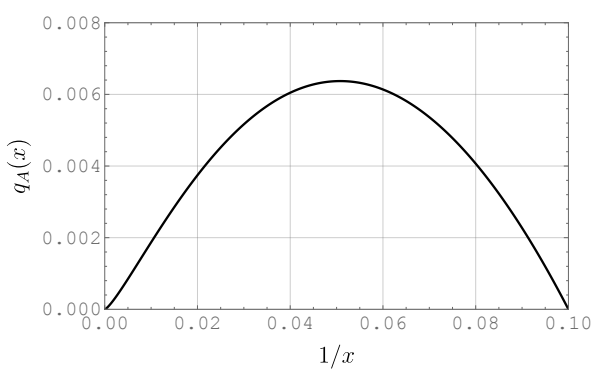

(a) $q_{A}(x)$

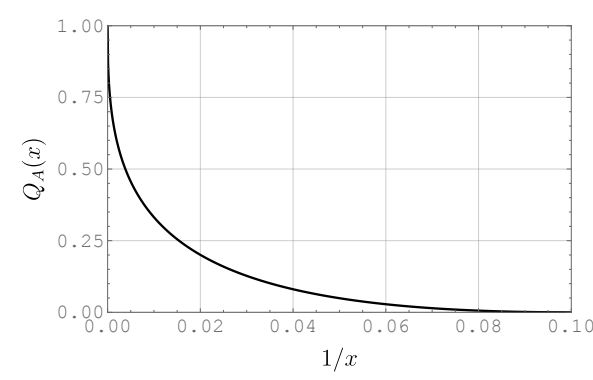

(b) $Q_{A}(x)$.

FIG. 7. Quasi-stationary distribution's $p d f q_{A}(x)$ and $c d f Q_{A}(x)$ as functions of $1 / x$ for $A=10$.

[7] P. Collet, S. Martínez, and J. S. Martín, Quasi-Stationary Distributions Markov Chains, Diffusions and Dynamical Systems, Probability and Its Applications, Springer, New York, NY, 2013.

[8] A. Comtet And C. Monthus, Diffusion in a one-dimensional random medium and hyperbolic Brownian motion, Journal of Physics A: Mathematical and General, 29 (1996), pp. 13311345, https://doi.org/10.1088/0305-4470/29/7/006.

[9] A. De Schepper And M. J. GoovaerTs, The GARCH(1,1)-M model: Results for the densities of the variance and the mean, Insurance: Mathematics and Economics, 24 (1999), pp. 8394, https://doi.org/10.1016/S0167-6687(98)00040-7.

[10] A. De Schepper, M. Teunen, and M. J. Goovaerts, An analytical inversion of a Laplace transform related to annuities certain, Insurance: Mathematics and Economics, 14 (1994), pp. 33-37, https://doi.org/10.1016/0167-6687(94)00004-2.

[11] C. Donati-Martin, R. Ghomrasni, And M. Yor, On certain Markov processes attached to exponential functionals of Brownian motion: Application to Asian options, Revista Matemática Iberoamericana, 17 (2001), pp. 179-193, https://doi.org/10.4171/RMI/292.

[12] D. Dufresne, The integral of geometric Brownian motion, Advances in Applied Probability, 33 (2001), pp. 223-241, https://doi.org/10.1239/aap/999187905.

[13] N. Dunford and J. T. Schwartz, Linear Operators. Part II: Spectral Theory. Self Adjoint Operators in Hilbert Space, John Wiley \& Sons, Inc., New York, NY, 1963.

[14] E. A. Feinberg and A. N. Shiryaev, Quickest detection of drift change for Brownian motion in generalized Bayesian and minimax settings, Statistics \& Decisions, 24 (2006), pp. 445470, https://doi.org/10.1524/stnd.2006.24.4.445.

[15] H. Geman And M. Yor, Bessel processes, Asian options, and perpetuities, Mathematical Finance, 3 (1993), pp. 349-375, https://doi.org/10.1111/j.1467-9965.1993.tb00092.x.

[16] I. S. Gradshteyn and I. M. Ryzhik, Table of Integrals, Series, and Products, Academic Press, eighth ed., 2014.

[17] K. Itô and H. P. McKean, JR., Diffusion Processes and Their Sample Paths, Springer, Berlin, Germany, 1974. 
[18] A. Kolmogonoff, Über die analitische Methoden in der Wahrscheinlichkeitsrechnung, Mathematische Annalen, 104 (1931), pp. 415-458, https://doi.org/10.1007/BF01457949. (in German).

[19] B. M. LEvitan, Eigenfunction expansions of second-order differential equations, Gostechizdat Moscow-Leningrad, Leningrad, USSR, 1950. (in Russian).

[20] B. M. Levitan AND I. S. SARgSjan, Introduction to Spectral Theory: Selfadjoint Ordinary Differential Operators, vol. 39 of Translations of Mathematical Monographs, American Mathematical Society, Providence, RI, 1975.

[21] V. Linetsky, Spectral expansions for Asian (average price) options, Operations Research, 52 (2004), pp. 856-867, https://doi.org/10.1287/opre.1040.0113.

[22] V. Linetsky, Spectral methods in derivative pricing, vol. 15 of Handbooks in Operations Research and Management Sciences, North-Holland, Netherlands, 2007, ch. 6, pp. 223-299.

[23] P. MANDL, Spectral theory of semi-groups connected with diffusion processes and its application, Czechoslovak Mathematical Journal, 11 (1961), pp. 558-569.

[24] S. Martínez AND J. S. Martín, Rates of decay and h-processes for one dimensional diffusions conditioned on non-absorption, Journal of Theoretical Probability, 14 (2001), pp. 199-212, https://doi.org/10.1023/A:1007881317492.

[25] S. Martínez ANd J. S. Martín, Classiffication of killed one-dimensional diffusions, Annals of Probability, 32 (2004), pp. 530-552, https://doi.org/10.1214/aop/1078415844.

[26] M. A. Milevsky, The present value of a stochasic perpetuity and the Gamma distribution, Insurance: Mathematics and Economics, 20 (1997), pp. 243-250, https://doi.org/10.1016/ S0167-6687(97)00013-9.

[27] C. Monthus And A. Comtet, On the flux distribution in a one dimensional disordered system, Journal de Physique I: France, 4 (1994), pp. 635-653, https://doi.org/10.1051/jp1:1994167.

[28] G. PESkIR, On the fundamental solution of the Kolmogorov-Shiryaev equation, in From Stochastic Calculus to Mathematical Finance: The Shiryaev Festschrift, Y. Kabanov, R. Liptser, and J. Stoyanov, eds., Springer, Berlin, 2006, pp. 535-546, https://doi.org/ 10.1007/978-3-540-30788-4_26.

[29] M. Pollak and D. Siegmund, A diffusion process and its applications to detecting a change in the drift of Brownian motion, Biometrika, 72 (1985), pp. 267-280, https://doi.org/10. 1093/biomet/72.2.267.

[30] A. S. Polunchenko, Asymptotic near-minimaxity of the randomized Shiryaev-Roberts-Pollak change-point detection procedure in continuous time, Theory of Probability and Its Applications, 64 (2017), pp. 769-786, https://doi.org/10.4213/tvp5142.

[31] A. S. Polunchenko, On the quasi-stationary distribution of the Shiryaev-Roberts diffusion, Sequential Analysis, 36 (2017), pp. 126-149, https://doi.org/10.1080/07474946.2016. 1275512.

[32] A. S. Polunchenko And G. Sokolov, An analytic expression for the distribution of the generalized Shiryaev-Roberts diffusion: The Fourier spectral expansion approach, Methodology and Computing in Applied Probability, 18 (2016), pp. 1153-1195, https://doi.org/10.1007/ s11009-016-9478-7.

[33] M. SchröDER, On the integral of geometric Brownian motion, Advances in Applied Probability, 35 (2003), pp. 159-183, https://doi.org/10.1239/aap/1046366104.

[34] A. N. ShIRYAEV, The problem of the most rapid detection of a disturbance in a stationary process, Soviet Mathematics-Doklady, 2 (1961), pp. 795-799. (Translated from Dokl. Akad. Nauk SSSR 138:1039-1042, 1961).

[35] A. N. ShiRYAev, On optimum methods in quickest detection problems, Theory of Probability and Its Applications, 8 (1963), pp. 22-46, https://doi.org/10.1137/1108002.

[36] A. N. SHIRYAEv, Quickest detection problems in the technical analysis of the financial data, in Mathematical Finance-Bachelier Congress 2000, H. Geman, D. Madan, S. R. Pliska, and T. Vorst, eds., Springer Finance, Springer, Berlin, 2002, pp. 487-521, https://doi.org/10. 1007/978-3-662-12429-1_22.

[37] L. J. Slater, Confluent Hypergeometric Functions, Cambridge University Press, Cambirdge, UK, 1960.

[38] E. C. Titchmarsh, Eigenfunction Expansions Associated with Second-Order Differential Equations, Clarendon, Oxford, UK, 1962.

[39] M. Vanneste, M. J. Goovaerts, and E. Labie, The distribution of annuities, Insurance: Mathematics and Economics, 15 (1994), pp. 37-48, https://doi.org/10.1016/0003-4916(88) 90283-7.

[40] E. T. WhitTAKer, An expression of certain known functions as generalized hypergeometric functions, Bulletin of the American Mathematical Society, 10 (1904), pp. 125-134.

[41] E. T. Whittaker and G. N. Watson, A Course of Modern Analysis, Cambridge University 
Press, fourth ed., 1927.

[42] E. Wong, The construction of a class of stationary Markoff processes, in Stochastic Processes in Mathematical Physics and Engineering, R. Bellman, ed., American Mathematical Society, Providence, RI, 1964, pp. 264-276.

[43] M. Yor, On some exponential functionals of Brownian motion, Advances in Applied Probability, 24 (1992), pp. 509-531. 